\title{
Rogue wave solutions for the infinite integrable nonlinear Schrödinger equation hierarchy
}

\author{
A. Ankiewicz and N. Akhmediev \\ Optical Sciences Group, Research School of Physics and Engineering, The Australian National University, Canberra, ACT 2600, Australia
}

(Received 19 May 2017; published 20 July 2017)

\begin{abstract}
We present rogue wave solutions of the integrable nonlinear Schrödinger equation hierarchy with an infinite number of higher-order terms. The latter include higher-order dispersion and higher-order nonlinear terms. In particular, we derive the fundamental rogue wave solutions for all orders of the hierarchy, with exact expressions for velocities, phase, and "stretching factors" in the solutions. We also present several examples of exact solutions of second-order rogue waves, including rogue wave triplets.
\end{abstract}

DOI: 10.1103/PhysRevE.96.012219

\section{INTRODUCTION}

The fundamental nonlinear Schrödinger equation (NLSE) is both integrable [1,2] and practically important in describing ocean waves [3,4], pulses in optical fibers [5,6] and dynamics of Bose-Einstein condensation [7]. Exact solutions to this equation include solitons [1], the Peregrine rogue wave [8], Akhmediev breathers [9,10], Kuznetsov-Ma solitons [11], and higher-order combinations of them [12]. These solutions are of practical use, with the most significant recent progress being in the theory of rogue waves [13-15].

Other integrable equations of comparable significance have also been found to have rogue wave solutions. Among them, we can mention the Davey-Stewartson equation [16], Hirota equation [17], Sasa-Satsuma equation [18], multicomponent NLSE [19], and many others. Various methods have been used to obtain related integrable equations. For example, the fourthorder Lakshmanan-Porsezian-Daniel (LPD) equation [20,21], its fifth-order extension [22], and others can be found due to their relation to certain types of moving helical space curves. This geometric analysis involves using unit tangent, normal, and binormal vectors, given by the Serret-Frenet equations, to describe a curve [23].

However, the equations considered in these works include only a finite number of higher-order terms. While this can be sufficient for specific applications, generalizations may become useful for further progress in this area. Recent work has extended analysis to the hierarchy of NLSEs with an infinite number of higher-order terms [24,25]. The latter describe higher-order dispersion and higher-order nonlinear terms that become important for waves with shorter duration. Integrability requires certain restrictions on the relative values of these terms at each order. However, each coefficient defining a particular order remains a free real parameter. As there is an infinite number of functionals in the hierarchy, there is an infinite number of free parameters. This fact provides a certain degree of flexibility in the exact solutions. Finding them is a challenge which has been addressed in [24,25]. In particular, soliton and breather solutions of the whole infinite hierarchy have been presented in explicit form.

Despite this evident progress, general rogue wave solutions in the presence of higher-order terms remain unknown. As rogue waves have usually short duration, the influence of higher-order terms is crucial for their formation. Here, we investigate this influence within the formalism of the integrable infinite hierarchy. This is not an easy task, and finding the complete solution that involves the whole infinite number of higher-order terms is possible only in the case of the lowestorder rogue wave solutions. Second-order rogue waves have been investigated in full only in the case of the fundamental NLSE. Here, we make progress in obtaining the second-order rogue wave solution when two coefficients are nonzero in the hierarchy. We consider this to be significant progress and hope to make further steps in future. As the interest in rogue waves is presently very high, we believe that future progress can be made with collective efforts.

The specific equation which we consider here is [24,25]

$$
i \psi_{x}+F[\psi(x, t)]=0,
$$

where the infinite length functional $F[\psi(x, t)]$ is

$$
F[\psi(x, t)]=\sum_{j=1}^{\infty}\left(\alpha_{2 j} K_{2 j}-i \alpha_{2 j+1} K_{2 j+1}\right)
$$

and where each coefficient $\alpha_{m}, m=2,3,4,5, \ldots, \infty$ is an arbitrary real number. In all expressions here, $x$ is the propagation variable and $t$ is the transverse variable (time in a moving frame), with the function $|\psi(x, t)|$ being the envelope of the waves. Thus, explicitly, the whole equation takes the form

$$
\begin{aligned}
i \psi_{x} & +\alpha_{2} K_{2}[\psi(x, t)]-i \alpha_{3} K_{3}[\psi(x, t)] \\
& +\alpha_{4} K_{4}[\psi(x, t)]-i \alpha_{5} K_{5}[\psi(x, t)] \\
& +\alpha_{6} K_{6}[\psi(x, t)]-i \alpha_{7} K_{7}[\psi(x, t)] \\
& +\alpha_{8} K_{8}[\psi(x, t)]-i \alpha_{9} K_{9}[\psi(x, t)]+\cdots=0 .
\end{aligned}
$$

For the lowest-order case, when all coefficients $\alpha_{j}$ except $\alpha_{2}$ are zero, we obtain the fundamental nonlinear Schrödinger equation:

$$
i \psi_{x}+\alpha_{2} K_{2}=i \psi_{x}+\alpha_{2}\left(\psi_{t t}+2 \psi|\psi|^{2}\right)=0
$$

Keeping additionally the third-order operator with nonzero $\alpha_{3}$, we obtain the Hirota equation [17]:

$$
i \psi_{x}+\alpha_{2} K_{2}-i \alpha_{3}\left[\psi_{t t t}+6|\psi|^{2} \psi_{t}\right]=0 .
$$

In the next stage, we keep $K_{4}$ as the fourth-order $(j=4)$ operator; it is often called the LPD operator [20,21]:

$$
\begin{aligned}
K_{4}[\psi(x, t)]= & \psi_{t t t t}+8|\psi|^{2} \psi_{t t}+6 \psi|\psi|^{4}+4 \psi\left|\psi_{t}\right|^{2} \\
& +6 \psi_{t}^{2} \psi^{*}+2 \psi^{2} \psi_{t t}^{*} .
\end{aligned}
$$


The fifth-order $(j=5)$, i.e., quintic, operator $K_{5}$ is

$$
\begin{aligned}
K_{5}[\psi(x, t)]= & \psi_{t t t t t}+10|\psi|^{2} \psi_{t t t}+10\left(\psi\left|\psi_{t}\right|^{2}\right)_{t} \\
& +20 \psi^{*} \psi_{t} \psi_{t t}+30|\psi|^{4} \psi_{t} .
\end{aligned}
$$

The sextic $K_{6}$ and heptic $K_{7}$ operators are given explicitly in [24]. The general form of the operators $K_{j}$ can be constructed recursively [25]. The complexity of these operators increases quickly with the order $j$. Nevertheless, the general form of the soliton and breather solutions can be derived explicitly [24]. Deriving the rogue wave solutions is more difficult. Here, for the first time, we present rogue wave solutions in general form.

Before starting, we provide some general observations. An important one is the scaling transformation. Namely, if we have a solution $\psi\left(x, t ; \alpha_{2}, \alpha_{3}, \alpha_{4}, \ldots\right)$ of the full equation, then we can generate a scaled solution by multiplying the function by an arbitrary real constant, $c$, multiplying $t$ by $c$, leaving $x$ unchanged, and multiplying each $\alpha_{j}$ in the solution by $c^{j}$. Hence the new solution is $c \psi\left(x, c t ; c^{2} \alpha_{2}, c^{3} \alpha_{3}, c^{4} \alpha_{4}, \ldots\right)$. So, the $\alpha_{j}$ 's in the solution are scaled, not the variable $x$. This applies to the solutions presented in this paper. This scaling is easy to apply, and so we use a unit background, $c=1$, in the solutions given here to maintain simplicity. Keeping this constant unit background in all solutions is the most convenient way to allow for comparison with existing results.

As can be seen from Eq. (3), the odd and even numbered functionals differ by a factor $i$ in front of each odd numbered functional. Then, naturally, equations containing odd and even numbered functionals have different properties. This means that the two types of equations should be considered separately. Following the notation of [24], we set $\alpha_{2 j}=0$ for every integer $j$ when considering solutions of the equation containing odd numbered functionals only, while we set $\alpha_{2 j+1}=0$ for every integer $j$ when considering solutions of the equation containing even numbered functionals only.

\section{Equations with odd numbered terms}

If all even-labeled coefficients are zero, i.e., $\alpha_{2 j}=0, j=$ $1,2,3, \ldots, \infty$, then, on canceling the common factor $i$, we obtain the equation which is infinite but contains only half of the terms of the original equation:

$$
\psi_{x}-\sum_{j=1}^{\infty} \alpha_{2 j+1} K_{2 j+1}=0 .
$$

These equations can have real-valued solutions. For example, if $\alpha_{3}$ is arbitrary and all other $\alpha_{j}^{\prime}$,'s are zero, then we obtain the "truncated" Hirota equation

$$
\psi_{x}-\alpha_{3}\left[\psi_{t t t}+6|\psi|^{2} \psi_{t}\right]=0
$$

which is sometimes called the "complex modified Kortewegde Vries (mKdV) equation". This is due to the obvious fact that any solution of the real $\mathrm{mKdV}$ equation,

$$
\psi_{x}-\alpha_{3}\left[\psi_{t t t}+6 \psi^{2} \psi_{t}\right]=0,
$$

will also satisfy Eq. (6) [26]. Rogue wave solutions of Eq. (6) have been given earlier in [27]. Indeed, this correspondence between complex and real solutions can be found for all odd numbered equations of the hierarchy. Let us start with the first-order rogue wave solutions. We should keep in mind that the order of the equation generating them remains infinite. The order of the solution and the order of the equation are different.

\section{FIRST-ORDER SOLUTIONS}

Below, we construct first-order solutions, step by step. First, we present solutions separately for equations containing odd or even numbered functionals only. Then we give the solutions for the general case where odd and even functionals in the equation are present simultaneously. This approach allows us to clearly see the features of rogue waves that are related to odd and even numbered terms in the equation.

\section{A. First-order solutions for equations containing the odd functionals only}

In this section, we allow for all odd operator coefficients $\alpha_{2 j+1}, j=1,2,3, \ldots, \infty$, to be arbitrary, while setting all even operator coefficients $\alpha_{2 j}, j=1,2,3, \ldots, \infty$, to zero. Then the solution is

$$
\psi(x, t)=\left[4 \frac{1+2 i B_{o} x}{D_{0}(x, t)}-1\right] \exp \left[i k\left(y_{0}+2 \phi_{o} x\right)\right],
$$

where

$$
D_{0}(x, t)=1+4 B_{o}^{2} x^{2}+4 y_{0}^{2},
$$

and $y_{0}=t+v_{o} x$, with $k$ being an arbitrary real number. The coefficient $v_{0}$ introduces tilt to the solution in the $(x, t)$ plane. Its action is similar to "velocity". Here $B_{o}, v_{o}$, and $\phi_{0}$ are obtained from summations (see the Appendices). $B_{o}$ can be related to Motzkin polynomial coefficients (given in Appendix A). Namely, we find

$$
\begin{aligned}
B_{o}= & 2 \sum_{n=1}^{\infty}(-1)^{n+1} n(2 n+1) k^{2 n-1} \alpha_{2 n+1} \\
& \times{ }_{2} F_{1}\left(\frac{1}{2}-n, 1-n ; 2 ;-\frac{4}{k^{2}}\right) \\
= & k\left[6 \alpha_{3}-20 \alpha_{5}\left(k^{2}-3\right)+42 \alpha_{7}\left(k^{4}-10 k^{2}+10\right)\right. \\
& -72 \alpha_{9}\left(k^{6}-21 k^{4}+70 k^{2}-35\right)+110 \alpha_{11} \\
& \left.\times\left(k^{8}-36 k^{6}+252 k^{4}-420 k^{2}+126\right)-\cdots\right],
\end{aligned}
$$

with ${ }_{2} F_{1}$ being a hypergeometric function [28].

The tilt coefficient $v_{0}$ in the above expressions is

$$
\begin{aligned}
v_{o}= & \sum_{n=1}^{\infty}(-1)^{n}(2 n+1) k^{2 n} \alpha_{2 n+12} F_{1}\left(\frac{1}{2}-n,-n ; 1 ;-\frac{4}{k^{2}}\right) \\
= & \sum_{n=1}^{\infty} v_{n}(k) \alpha_{2 n+1} \\
= & -3 \alpha_{3}\left(k^{2}-2\right)+5 \alpha_{5}\left(k^{4}-12 k^{2}+6\right)-7 \alpha_{7}\left(k^{6}-30 k^{4}\right. \\
& \left.+90 k^{2}-20\right)+9 \alpha_{9}\left(k^{8}-56 k^{6}+420 k^{4}-560 k^{2}+70\right) \\
& -11 \alpha_{11}\left(k^{10}-90 k^{8}+1260 k^{6}-4200 k^{4}\right. \\
& \left.+3150 k^{2}-252\right)+\cdots,
\end{aligned}
$$




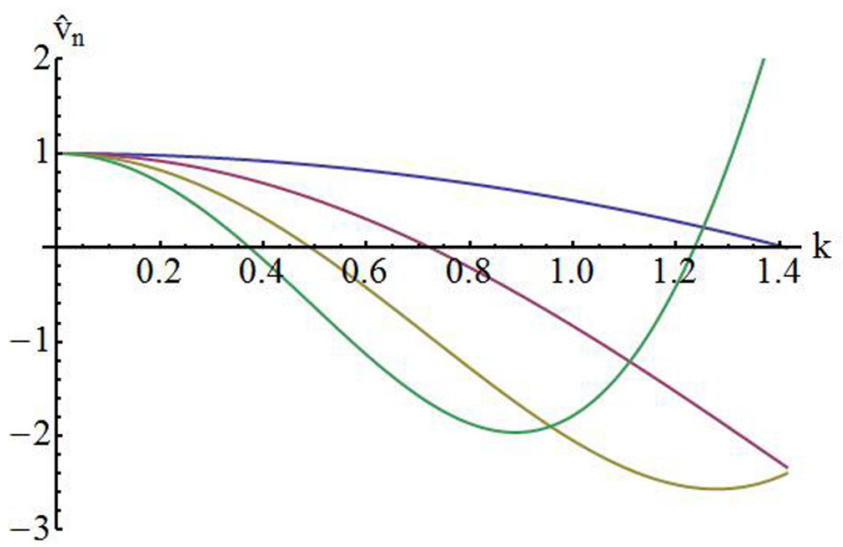

FIG. 1. Plot of relative factors $\hat{v}_{n}(k)$ as a function of $k$ calculated using Eqs. (11), (12), and (14), for $n=1$ (uppermost curve, blue), $n=2$ (second curve from top, magenta), $n=3$ (third curve from top, olive), and $n=4$ (lowest curve, green).

while the phase coefficient $\phi_{0}$ is given by

$$
\begin{aligned}
\phi_{o}= & \sum_{n=1}^{\infty}(-1)^{n+1} n k^{2 n} \alpha_{2 n+1}{ }_{2} F_{1}\left(-n-\frac{1}{2}, 1-n ; 1 ;-\frac{4}{k^{2}}\right) \\
= & k^{2}\left[\alpha_{3}-2 \alpha_{5}\left(k^{2}-10\right)+3 \alpha_{7}\left(k^{4}-28 k^{2}+70\right)\right. \\
& -4 \alpha_{9}\left(k^{6}-54 k^{4}+378 k^{2}-420\right)+5 \alpha_{11}\left(k^{8}-88 k^{6}\right. \\
& \left.\left.+1188 k^{4}-3696 k^{2}+2310\right)-\cdots\right] .
\end{aligned}
$$

For convenience, we define relative values of the factors in Eq. (12) as

$$
\hat{v}_{n}(k)=\frac{v_{n}(k)}{v_{n}(0)},
$$

These are given by

$$
\hat{v}_{1}=1-\frac{k^{2}}{2}, \quad \hat{v}_{2}=1-2 k^{2}+\frac{k^{4}}{6},
$$

etc. These relative factors are plotted in Fig. 1. From Fig. 1, we can see that, when there is only a single nonzero $\alpha_{2 n+1}$, then the tilt factor, $\hat{v}_{n}(k)$, can be zero at some values of $k$. Consequently, a rogue wave for an equation with odd numbered terms can have a symmetric intensity pattern.

If $k=0$, then only $v_{0}$ enters into the solution, as the other two components are zero. We then get soliton-type solutions found earlier in [24]. These real-valued solutions are

$$
\psi(x, t)=\left[\frac{4}{1+4\left(t+v_{o} x\right)^{2}}-1\right]
$$

with $v_{0}=\sum_{n=1}^{\infty} \frac{(2 n+1) !}{(n !)^{2}} \alpha_{2 n+1}$, as given in [24]. This can be viewed as an extension of the algebraic soliton of $\mathrm{mKdV}$ related equations. Then, along the diagonal line $t+v_{o} x=$ 0 , we have a constant value, $\psi(x, t)=3$. Consequently, this solution looks like a moving soliton on a background. It is not localized in two dimensions like a "true" rogue wave. When $k$ is not zero, the resulting solutions are similar to NLSE rogue waves, apart from the fact that the central part is tilted in the $(x, t)$ plane. One example is given in Fig. 2. In Fig. 3 we give another example where the tilt is zero. Here, only $\alpha_{3}$ is

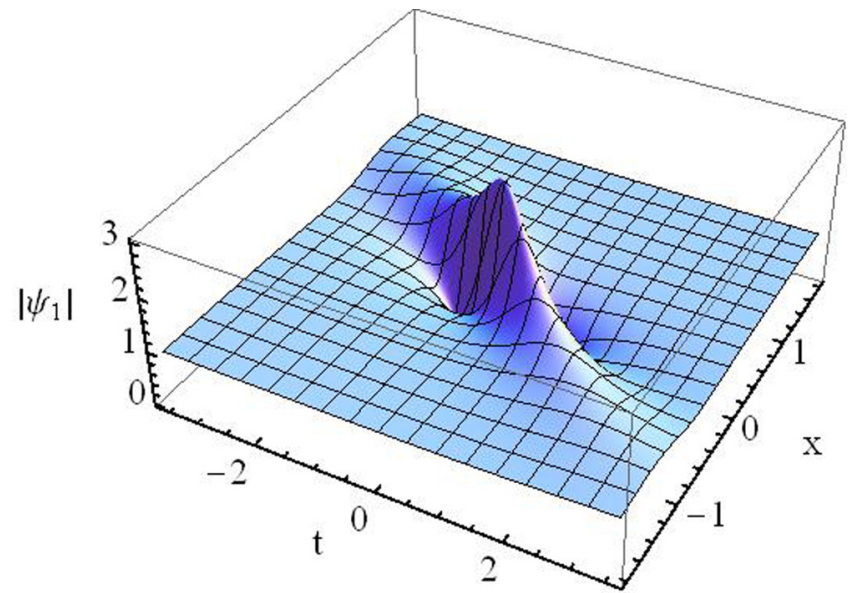

FIG. 2. Plot of the first-order rogue wave, defined by Eq. (8). The two coefficients in Eq. (3) are $\alpha_{3}=\frac{1}{2}$ and $\alpha_{5}=-\frac{1}{32}$ while all other $\alpha_{j}$ 's zero. Here $k=1$.

nonzero, and we take $k=\sqrt{2}$ to set $v_{0}=0$ in Eq. (11). This choice leads to a nontilted intensity pattern.

\section{B. First-order solutions for equations containing the even functionals only}

The cases of even functionals and rogue wave solutions for them have been considered in [24]. However, the solutions in [24] were not fully general, as they lack tilt parts. We now extend the even functional rogue waves of [24] to allow for a velocity, so that the waveform is tilted. We find

$$
\psi(x, t)=\left[4 \frac{1+2 i B_{e} x}{D_{e}(x, t)}-1\right] \exp \left[i\left(k y_{e}+\phi_{e} x\right)\right],
$$

where

$$
D_{e}(x, t)=1+4 B_{e}^{2} x^{2}+4 y_{e}^{2},
$$

and $y_{e}=t+v_{e} x$, with $k$ being an arbitrary real number.

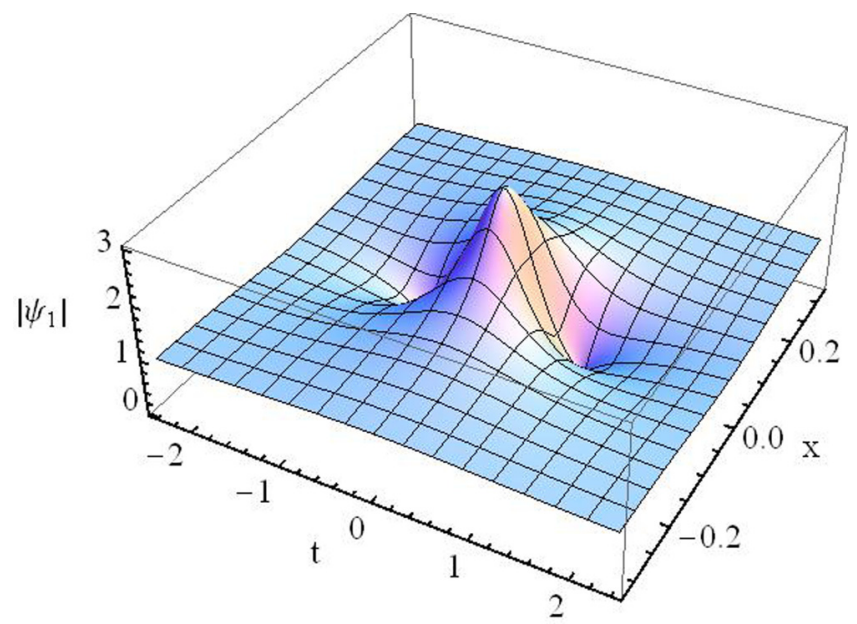

FIG. 3. Plot of the first-order rogue wave defined by Eq. (8). The coefficient $\alpha_{3}=1$. All other $\alpha_{j}$ 's in Eq. (3) are zero. Here $k=\sqrt{2}$, giving $\hat{v}_{1}=0$ as can be seen from Fig. 1 . Hence, $v_{0}=0$. This choice leads to a symmetric intensity pattern. 
Here,

$$
\begin{aligned}
B_{e}= & 2 \sum_{n=1}^{\infty}(-1)^{n+1} n(2 n-1) \alpha_{2 n} k^{2 n-2} \\
& \times{ }_{2} F_{1}\left(1-n, \frac{3}{2}-n ; 2 ;-\frac{4}{k^{2}}\right) \\
= & 2 \alpha_{2}-12 \alpha_{4}\left(k^{2}-1\right)+30 \alpha_{6}\left(k^{4}-6 k^{2}+2\right) \\
& -56 \alpha_{8}\left(k^{6}-15 k^{4}+30 k^{2}-5\right)+90 \alpha_{10}\left(k^{8}-28 k^{6}\right. \\
& \left.+140 k^{4}-140 k^{2}+14\right)-\cdots, \\
\phi_{e}= & \sum_{n=1}^{\infty}(-1)^{n+1} \alpha_{2 n} k^{2 n}(2 n-1){ }_{2} F_{1}\left(\frac{3}{2}-n,-n ; 1 ;-\frac{4}{k^{2}}\right) \\
= & \alpha_{2}\left(k^{2}+2\right)-3 \alpha_{4}\left(k^{4}-4 k^{2}-2\right)+5 \alpha_{6}\left(k^{6}-18 k^{4}\right. \\
& \left.+18 k^{2}+4\right)-7 \alpha_{8}\left(k^{8}-40 k^{6}+180 k^{4}-80 k^{2}-10\right) \\
& +9 \alpha_{10}\left(k^{10}-70 k^{8}+700 k^{6}-1400 k^{4}+350 k^{2}+28\right) \\
& -\cdots,
\end{aligned}
$$

and

$$
\begin{aligned}
v_{e}= & 2 \sum_{n=1}^{\infty}(-1)^{n} n \alpha_{2 n} k_{2 n-1}^{2 n} F_{1}\left(\frac{1}{2}-n, 1-n ; 1 ;-\frac{4}{k^{2}}\right) \\
= & k\left[-2 \alpha_{2}+4 \alpha_{4}\left(k^{2}-6\right)-6 \alpha_{6}\left(k^{4}-20 k^{2}+30\right)\right. \\
& +8 \alpha_{8}\left(k^{6}-42 k^{4}+210 k^{2}-140\right)-10 \alpha_{10}\left(k^{8}-72 k^{6}\right. \\
& \left.\left.+756 k^{4}-1680 k^{2}+630\right)+\cdots\right] .
\end{aligned}
$$

As an example, we can take $\alpha_{4} \neq 0$ but all other $\alpha$ 's are zero. Then if we take $k= \pm \sqrt{6}$, we have a symmetric intensity pattern, as $v_{e}=0$, but the phase is still tilted due to the factor $\exp \left[i\left( \pm \sqrt{6} t-30 \alpha_{4} x\right)\right]$.

If $k=0$, then

$$
\begin{aligned}
\phi_{e} & =\sum_{n=1}^{\infty} \frac{(2 n) !}{(n !)^{2}} \alpha_{2 n} \\
& =2\left(\alpha_{2}+3 \alpha_{4}+10 \alpha_{6}+35 \alpha_{8}+126 \alpha_{10}+\cdots\right),
\end{aligned}
$$

and

$$
\begin{aligned}
B_{e} & =\sum_{n=1}^{\infty} \frac{n(2 n) !}{(n !)^{2}} \alpha_{2 n} \\
& =2\left(\alpha_{2}+6 \alpha_{4}+30 \alpha_{6}+140 \alpha_{8}+630 \alpha_{10}+\cdots\right) .
\end{aligned}
$$

This $k=0$ special case of the solution is the zero-velocity case for equations containing only even numbered functionals; it was found earlier in [24].

We note that, for the even functionals, these $k=0$ solutions [involving Eqs. (20) and (21)] are still fully localized. This contrasts with the odd functional $k=0$ solutions which still contain a superimposed "soliton" when far from the central part.

\section{General first-order rogue wave formula}

Having in hand the polynomials found separately for even and odd functionals in the above sections, we are now in a position to present a general first-order solution for the partial differential equation (PDE) containing an infinite number of functionals, where each real coefficient, $\alpha_{2}, \alpha_{3}, \alpha_{4}, \alpha_{5}, \ldots$ is arbitrary. Namely, this general solution can be written as

$$
\psi(x, t)=\left[4 \frac{1+2 i B_{s} x}{D_{s}(x, t)}-1\right] \exp \left[i\left(k y_{s}+\phi_{s} x\right)\right],
$$

where $B_{s}=B_{e}+B_{o}$ with $B_{o}$ and $B_{e}$ given by Eqs. (10) and (17), respectively, while

$$
D_{s}(x, t)=1+4 B_{s}^{2} x^{2}+4 y_{s}^{2}
$$

with $y_{s}=t+v_{s} x$ and $v_{s}=v_{0}+v_{e}$, which is composed of $v_{o}$ and $v_{e}$ given by Eqs. (11) and (19), respectively. The phase coefficient $\phi_{s}=\phi_{e}+2 k \phi_{o}$, where $\phi_{o}$ and $\phi_{e}$ are given by Eqs. (13) and (18), respectively.

We stress that Eq. (22) is the general first-order rogue wave solution for Eq. (1), which includes an infinite number of terms. Any particular case can be obtained from Eq. (22) by taking specific values of coefficients $\alpha_{j}$ in the hierarchy and solution parameter, $k$.

\section{SECOND-ORDER SOLUTIONS}

\section{A. Real valued second-order solutions for odd-order equations}

After having the general first-order rogue wave solution, we now turn our attention to the second-order solutions. Again, first, we consider real-valued second-order solutions. These are significantly simpler than the complex ones. Also, their importance is similar to that of the $\mathrm{mKdV}$ equation. So, for this section, we are taking $|\psi|=\psi$ in all operators $K_{2 j+1}$ in the full equation. If we take $j=1$ only, thus setting $\alpha_{3} \neq 0$ and $\alpha_{2 j+1}=0$ for $j \neq 1$, then we deal with the truncated Hirota equation (6) which reduces to the (real) $\mathrm{mKdV}$ equation (7). For this special case only, the second-order solution was given, with a different format, in [26]. Here, we provide the general solution when all odd-indexed coefficients can be nonzero. This can be done by using the form of the third-order equation result and finding the pattern of coefficients needed for the velocity and stretching factor terms of higher-order solutions.

The general real solution, for any combination of odd-order operators, is given by

$$
\psi_{2}=1+12 \frac{G_{2}}{D_{2}},
$$

where

$$
\begin{aligned}
G_{2}= & 3-8 y\left(3 y+2 y^{3}+8 x B_{s}\right), \\
D_{2}= & 9+108 y^{2}+48 y^{4}+64 y^{6} \\
& +64 x B_{s}\left(3 y-4 y^{3}+4 x B_{s}\right) .
\end{aligned}
$$

The variable $y$ in the above expressions is $y=t+v_{m} x$. The factor $y$ appears several times in the solution. The tilt coefficient $v_{m}$ is contained within $y$. It not directly a velocity:

$$
\begin{aligned}
v_{m} & =\sum_{n=1}^{\infty} \frac{(2 n+1) !}{(n !)^{2}} \alpha_{2 n+1} \\
& =6 \alpha_{3}+30 \alpha_{5}+140 \alpha_{7}+630 \alpha_{9}+2772 \alpha_{11}+\cdots .
\end{aligned}
$$




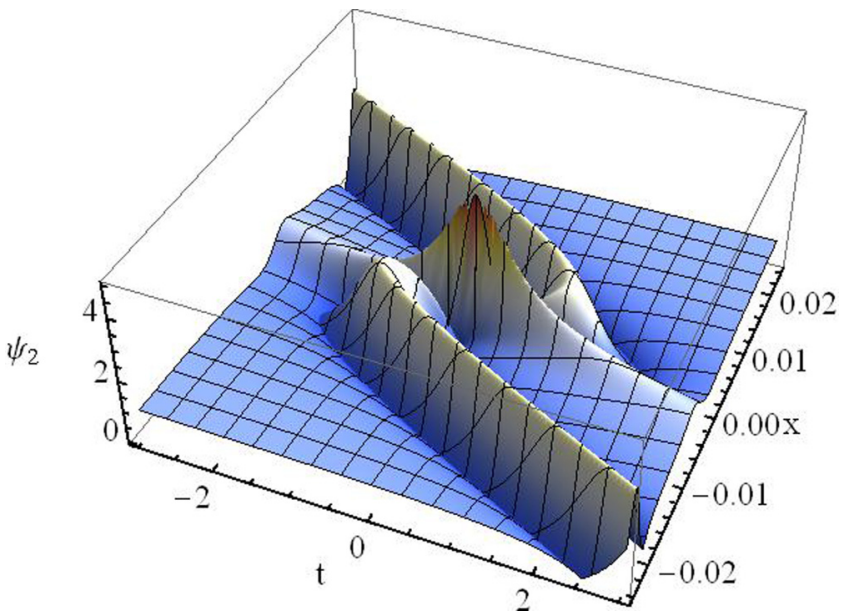

FIG. 4. Plot of the second-order real solution, $\psi_{2}$, given by Eq. (24). The coefficients of Eq. (1) are $\alpha_{3}=1, \alpha_{5}=1 / 2, \alpha_{7}=1 / 4$, $\alpha_{9}=1 / 8$, and $\alpha_{11}=1 / 16$.

Furthermore, the stretching factor is

$$
\begin{aligned}
B_{s} & =\sum_{n=1}^{\infty} n \frac{(2 n+1) !}{(n !)^{2}} \alpha_{2 n+1}, \\
& =6 \alpha_{3}+60 \alpha_{5}+420 \alpha_{7}+2520 \alpha_{9}+13860 \alpha_{11}+\cdots .
\end{aligned}
$$

In order to demonstrate that the equation with an infinite number of terms can be solved, we can specify the infinite set of coefficients $\alpha_{2 n+1}$ by a single expression. This can lead us to a closed form expression for the solution of the equation with an infinite number of terms. For example, let us take $\alpha_{2 n+1}=1 /(2 n+1)$ !. In this case, the tilt factor $v_{m}=I_{0}(2)-$ $1 \approx 1.2796$ and the stretching factor is $B_{s}=I_{1}(2) \approx 1.5906$, with $I_{0}$ and $I_{1}$ being modified Bessel functions.

Any other case with a finite number of terms can be considered individually. We plot an example in Fig. 4 for a case where five coefficients $\alpha_{j}$ are nonzero. In all these cases, the wave is not entirely localized, and the structure resembles a rogue wave on top of two interacting solitons. We can interpret this shape by saying that the interaction between two algebraic solitons produces a rogue wave in the center. The central maximum has always the height 5 for a background of 1. Remarkably, this is the same amplitude as for the second-order NLSE rogue wave. The significance of this fact will be considered elsewhere. For a simpler case, when only $\alpha_{3}$ is nonzero, the result of Eq. (24) reduces to that given for the $\mathrm{mKdV}$ in [26]. Again, here, we present the most general second-order solution of this type.

\section{B. The solution with an additional free parameter}

In a study of the rogue waves of the Hirota equation [27], it was pointed out that a free parameter can be included in the solution (thus making it complex). In our present notations, this applies when only $\alpha_{3}$ is nonzero. Such a free parameter can be also added to the present solutions. Then, the whole infinite equation with the odd-order terms has the following solution:

$$
\psi_{2}=1+12 \frac{G_{2}+i H_{2}}{\bar{D}_{2}},
$$

where $G_{2}, v_{m}, B_{s}$, and $y=t+v_{m} x$ are as above given by Eqs. (25)-(28), while

$$
H_{2}=k_{s}\left(1+4 y^{2}\right)
$$

and

$$
\begin{aligned}
\bar{D}_{2}= & D_{2}+k_{s}^{2}=9+k_{s}^{2}+108 y^{2}+48 y^{4}+64 y^{6} \\
& +64 x B_{s}\left(3 y-4 y^{3}+4 x B_{s}\right)
\end{aligned}
$$

contain a free parameter $k_{s}$. We note that the solution given by Eq. (29) is now complex but it does not include an exponential factor.

\section{Second-order solutions for equations with even-order coefficients}

Increasing the complexity of the hierarchy step by step, we now consider an equation with a single even-order coefficient, i.e.,

$$
i \psi_{x}+\alpha_{2 j} K_{2 j}=0,
$$

with a fixed but arbitrary value of the integer $j$. The secondorder rogue wave solution for this case is

$$
\psi_{2}^{(j)}=\left(1+12 \frac{G_{j}+i H_{j}}{D_{j}}\right) e^{i \phi_{j} x}
$$

where

$$
\begin{aligned}
& G_{j}=3-2(8 j+1) X^{2}-T^{4}-6 T^{2}\left(X^{2}+1\right)-5 X^{4}, \\
& H_{j}=-X\left[2 T^{2}\left(X^{2}+1-4 j\right)+2(4 j-3) X^{2}\right. \\
&\left.-8 j+T^{4}+X^{4}-7\right], \\
& D_{j}= 3 T^{2}\left[X^{4}-2(8 j-5) X^{2}+9\right] \\
&+(16 j+11) X^{4}+\left[(8 j+1)^{2}+18\right] X^{2} \\
&+T^{6}+3 T^{4}\left(X^{2}+1\right)+X^{6}+9,
\end{aligned}
$$

with $X=2 x B_{j}, T=2 t$. Here the stretching factor relates to the phase as follows:

$$
B_{j}=j \phi_{j}=\frac{j(2 j) !}{(j !)^{2}} \alpha_{2 j} .
$$

We plot an example of this solution in Fig. 5 for the case $j=$ 4 with $\alpha_{8}=1 / 60$. The solution resembles the second-order rogue wave solution of the NLSE. However, the details of this shape depend on the value of $j$.

\section{The solution when $\alpha_{2}$ and $\alpha_{4}$ are both nonzero}

If we have only the functionals $K_{2}$ and $K_{4}$ included into the equation, i.e.,

$$
i \psi_{x}+\alpha_{2} K_{2}+\alpha_{4} K_{4}=0,
$$




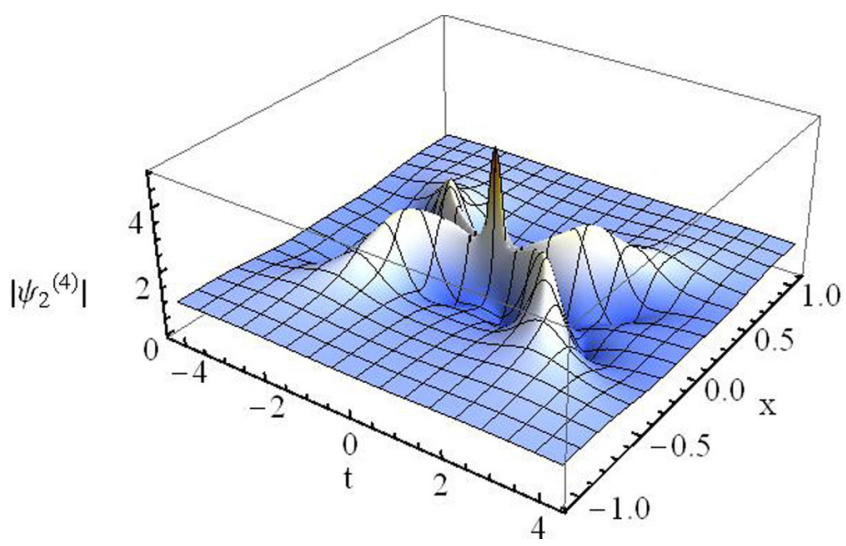

FIG. 5. Second-order complex solution, with $j=4$, i.e., $\psi_{2}^{(4)}$ from Eqs. (33)-(36). Here $\alpha_{8}=1 / 60$ (and all other $\alpha^{\prime}$ s are zero).

with $\alpha_{2}$ and $\alpha_{4}$ being arbitrary real numbers, and all other $\alpha_{j}$ 's being zero, then the solution takes the form

$$
\psi_{2}^{(2,4)}=\left(1+12 \frac{G_{2,4}+i H_{2,4}}{D_{2,4}}\right) e^{i 2\left(\alpha_{2}+3 \alpha_{4}\right) x}
$$

where

$$
G_{2,4}=3-T^{4}-6 T^{2}\left(X^{2}+1\right)-24\left(3 \alpha_{2}+34 \alpha_{4}\right) x X-5 X^{4},
$$

$$
\begin{aligned}
H_{2,4}= & -X\left[T^{4}+2 T^{2}\left(X^{2}-7\right)+8\left(\alpha_{2}+30 \alpha_{4}\right) x X\right. \\
& \left.+X^{4}-23\right]-32 \alpha_{2}\left(T^{2}+1\right) x \\
D_{2,4}= & 3 T^{2}\left[9-24\left(\alpha_{2}+22 \alpha_{4}\right) x X+X^{4}\right] \\
& +144\left(11 \alpha_{2}^{2}+228 \alpha_{2} \alpha_{4}+1228 \alpha_{4}^{2}\right) x^{2}+3 T^{4}\left(X^{2}+1\right) \\
+ & 12\left(9 \alpha_{2}+86 \alpha_{4}\right) x X^{3}+X^{6}+9+T^{6}
\end{aligned}
$$

with $X=2 x B_{s}, T=2 t$, and the stretching factor is $B_{s}=$ $2 \alpha_{2}+12 \alpha_{4}$. This result, given by Eqs. (38)-(41), generalizes that given in Appendix B of [29].

\section{E. Triplet solutions}

One important aspect of higher-order rogue waves is their ability to split into elementary components [30]. The latter are normally the first-order rogue waves of the same equation [31]. The splitting requires additional free parameters in the solution. Using the technique of [30] and adding two more arbitrary real parameters, $\beta$ and $\gamma$, we obtain a more general second-order solution than that given by Eqs. (33)-(36). Namely, for any positive integer $j$, we have

$$
\hat{\psi}_{2}^{(j)}=\left(1+12 \frac{\hat{G}_{j}+i \hat{H}_{j}}{\hat{D}_{j}}\right) e^{i \phi_{j} x}
$$

where

$$
\begin{gathered}
\hat{G}_{j}=G_{j}-2 \beta T+2 \gamma X, \\
\hat{H}_{j}=H_{j}-2 \beta T X+\gamma\left(X^{2}-T^{2}-1\right), \\
\hat{D}_{j}=D_{j}+\beta\left[\beta+2 T\left(3 X^{2}-T^{2}+3\right)\right] \\
+\gamma\left[\gamma+2 X\left(3 T^{2}-X^{2}-8 j-1\right)\right],
\end{gathered}
$$

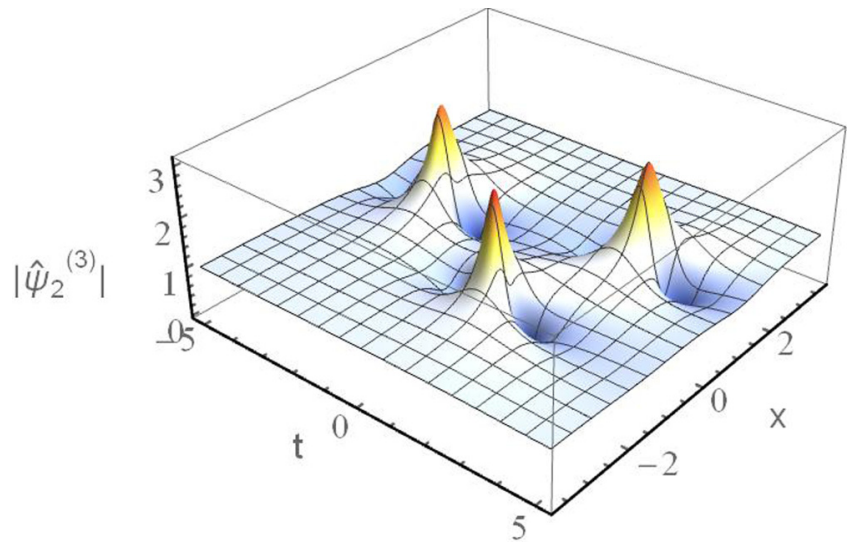

FIG. 6. Second-order triplet solution $\left|\hat{\psi}_{2}^{(3)}\right|$, with $j=3$, which is defined by Eqs. (42)-(45). Here $\alpha_{6}=0.03$ (and all other $\alpha$ 's are zero), while additional parameters $\beta=-130$ and $\gamma=-180$.

where $G_{j}, H_{j}$, and $D_{j}$ are given in Eqs. (34)-(36) and $X=2 x B_{j}, T=2 t$. Further, $B_{j}=j \phi_{j}=\frac{j(2 j) !}{(j !)^{2}} \alpha_{2 j}$, as before. These solutions apply when $\alpha_{2 j}$ is the only nonzero coefficient. If $\beta=\gamma=0$, then these solutions reduce to those given by Eqs. (33)-(36), i.e., $\hat{\psi}_{2}^{(j)}$ reduces to $\psi_{2}^{(j)}$. When $j=1$, we recover the known triplet solution given in [30,32,33]. We plot an example of a triplet solution in Fig. 6 for the case $j=3$ with $\alpha_{6}=0.03$.

\section{CONCLUSION}

We have presented new rogue wave solutions for the equations of the infinite integrable NLSE hierarchy. The equations of this hierarchy have various properties contributed by the even and odd numbered functionals. Consequently, the properties of rogue wave solutions differ, depending on whether even or odd numbered functionals are present. Therefore, it is important to consider their roles separately before the general solution is derived. Following this principle, we have derived a first-order rogue wave solution for three cases: separately for equations with even and odd numbered functionals, and then the most general solution when all functionals of the infinite equation can be present. All particular solutions can be deduced from the latter case.

The same principle can be applied for the second- and higher-order rogue wave solutions. However, these are significantly more complicated. We have given some examples of second-order solutions for special cases of the infinite NLSE hierarchy, leaving further progress for future publications. However, even these special cases provide general ideas on what the second-order rogue wave solutions look like.

The infinite NLSE hierarchy contains higher-order terms that appear in applications. These include higher-order dispersion terms and higher-order nonlinear terms. They emerge in equations both for waves in optical fibers [6] and for ocean waves [34-36] when the wave duration becomes shorter. This limit is certainly relevant for rogue waves. Clarifying the roles of these terms in the formation of rogue waves is an important practical issue. Despite the fact that the requirement of integrability imposes limitations, they can be resolved in 
TABLE I. The nonzero Motzkin coefficients, $M_{n, r}$, for $0 \leqslant r \leqslant$ $n-1$.

\begin{tabular}{|c|c|c|c|c|c|c|}
\hline$n$ & \multicolumn{6}{|c|}{ Motzkin coefficients, $M_{n, r}$} \\
\hline 1 & 1 & & & & & \\
\hline 2 & 1 & 3 & & & & \\
\hline 3 & 1 & 10 & 10 & & & \\
\hline 4 & 1 & 21 & 70 & 35 & & \\
\hline 5 & 1 & 36 & 252 & 420 & 126 & \\
\hline 6 & 1 & 55 & 660 & 2310 & 2310 & 462 \\
\hline
\end{tabular}

a step-by-step approach to the problem. Our present paper is one of these necessary steps.

\section{ACKNOWLEDGMENTS}

The authors acknowledge the support of the Australian Research Council (Discovery Projects No. DP140100265 and No. DP150102057) and support from the Volkswagen Stiftung.

\section{APPENDIX A: DERIVATION OF $B_{0}$}

In these Appendices, we provide a way of deriving the coefficients that appear in the solutions. In order to obtain the pattern of the solution coefficients needed, we substitute directly for a few lower-order equations and observe their form.

To take the $B_{0}$ derivation as an example, we find that

$$
B_{o}=k\left[6 \alpha_{3}-20 \alpha_{5}\left(k^{2}-3\right)+42 \alpha_{7}\left(k^{4}-10 k^{2}+10\right)+\cdots\right] \text {. }
$$

In fact, the polynomial multiplying the term with $\alpha_{2 n+1}$ takes the form

$$
(2 n+1) !(-1)^{n+1} k^{2 n-1} \sum_{r=0}^{n} \frac{(-1)^{r} k^{-2 r}}{(r+1) ! r !(2 n-2 r-1) !} .
$$

We note that there is a geometric link, in that the number of ways of drawing $r$ nonintersecting lines between $2 n-1$ points on a circle is $M_{n, r}=\frac{(2 n-1) !}{(r+1) ! r !(2 n-2 r-1) !}$, i.e., they are Motzkin polynomial coefficients. In fact Motzkin numbers and polynomials [37] can be useful in counting objects in combinatorics. They are listed in Table I.

It is plain that these numbers are found, with alternating signs, in the coefficients of $B_{0}$. Here, for a given number, $n$, these $n$ numbers in the second column appear with the term $\alpha_{2 n+1}$ [see Eq. (A1)]. The premultiplying factor is $2(-1)^{n+1} n(2 n+1)$.

The convenient point is that this can be summed to give a closed form result for the factor with coefficient $\alpha_{2 n+1}$, viz.,

$$
2(-1)^{n+1} n(2 n+1) k_{2}^{2 n-1} F_{1}\left(\frac{1}{2}-n, 1-n ; 2 ;-\frac{4}{k^{2}}\right),
$$

thus leading to Eq. (10) in the text.

\section{APPENDIX B: DERIVATION OF $v_{0}$}

To take the $v_{0}$ derivation as another example, we find that

$$
v_{o}=-3\left(-2+k^{2}\right) \alpha_{3}+5\left(6-12 k^{2}+k^{4}\right) \alpha_{5}+\cdots \text {. }
$$

TABLE II. The nonzero Motzkin coefficients, $Q_{n, r}$, for $0 \leqslant r \leqslant$ $n-1$.

\begin{tabular}{lllllll}
\hline \hline$n$ & \multicolumn{1}{l}{ Motzkin coefficients, $Q_{n, r}$} & & \\
\hline 1 & 1 & & & & & \\
2 & 1 & 1 & & & & \\
3 & 1 & 6 & 2 & & \\
4 & 1 & 15 & 30 & 5 & 14 & \\
5 & 1 & 28 & 140 & 140 & 630 & 42 \\
6 & 1 & 45 & 420 & 1050 & \\
\hline \hline
\end{tabular}

In fact, the polynomial multiplying the term with $\alpha_{2 n+1}$ takes the form

$$
(2 n+1) !(-1)^{n} k^{2 n} \sum_{r=0}^{n} \frac{(-1)^{r} k^{-2 r}}{(r !)^{2}(2 n-2 r) !} .
$$

The convenient point is that this can be summed to give a closed form result for the factor with coefficient $\alpha_{2 n+1}$, viz.,

$$
(-1)^{n}(2 n+1) k_{2}^{2 n} F_{1}\left(\frac{1}{2}-n,-n ; 1 ;-\frac{4}{k^{2}}\right),
$$

thus leading to Eq. (11) in the text.

\section{APPENDIX C: DERIVATION OF $B_{e}$}

We now take the $B_{e}$ derivation as a further example. We find that

$$
\begin{aligned}
B_{e}= & 2 \alpha_{2}-12 \alpha_{4}\left(k^{2}-1\right)+30 \alpha_{6}\left(k^{4}-6 k^{2}+2\right) \\
& -56 \alpha_{8}\left(k^{6}-15 k^{4}+30 k^{2}-5\right)+\cdots .
\end{aligned}
$$

Here, the polynomial multiplying the term with $\alpha_{2 n}$ takes the form

$$
(2 n) !(-1)^{n+1} k^{2 n-2} \sum_{r=0}^{n} \frac{(-1)^{r} k^{-2 r}}{(r+1) ! r !(2 n-2 r-2) !} .
$$

Related to the earlier result, the number of ways of drawing $r$ nonintersecting lines between $2 n-2$ points on a circle is $Q_{n, r}=\frac{(2 n-2) !}{(r+1) ! r !(2 n-2 r-2) !}$, i.e., they are another set of Motzkin polynomial coefficients and are listed in Table II.

Clearly, these numbers occur in the coefficients of $B_{e}$ [see Eq. (C1)]. Here, for a given number, $n$, these $n$ numbers in the second column appear with alternating signs, with the term $\alpha_{2 n}$ [see Eq. (17)]. The premultiplying factor is $2(-1)^{n+1} n$ $(2 n-1)$.

Again, this can be summed to give a closed form result for the factor with coefficient $\alpha_{2 n+1}$, viz.,

$$
2(-1)^{n+1} n(2 n-1) k_{2}^{2 n-2} F_{1}\left(1-n, \frac{3}{2}-n ; 2 ;-\frac{4}{k^{2}}\right),
$$

thus leading to Eq. (17) in the main text.

Then, the coefficients $v_{e}, \phi_{0}$, and $\phi_{e}$ can be found in similar ways. 
[1] V. E. Zakharov and A. B. Shabat, Exact theory of twodimensional self-focusing and one-dimensional self-modulation of waves in nonlinear media, Zh. Eksp. Teor. Fiz. 61, 118 (1971) [J. Exp. Theor. Phys. 34, 62 (1972)].

[2] N. Akhmediev and A. Ankiewicz, Solitons, Nonlinear Pulses and Beams (Chapman and Hall, London, 1997).

[3] V. E. Zakharov, Stability of periodic waves of finite amplitude on a surface of deep fluid, J. Appl. Mech. Tech. Phys. 9, 190 (1968).

[4] C. Kharif, E. Pelinovsky, and A. Slunyaev, Rogue Waves in the Ocean (Springer, Berlin, 2009).

[5] A. Hasegawa and F. Tappert, Transmission of stationary nonlinear optical pulses in dispersive dielectric fibers. I. Anomalous dispersion, Appl. Phys. Lett. 23, 142 (1973).

[6] G. P. Agrawal, Nonlinear Fiber Optics (Optics and Photonics), 4th ed. (Elsevier, Amsterdam, 2006).

[7] P. A. Andreev, First principles derivation of NLS equation for BEC with cubic and quintic nonlinearities at nonzero temperature: Dispersion of linear waves, Int. J. Mod. Phys. B 27, 1350017 (2013).

[8] D. H. Peregrine, Water waves, nonlinear Schrödinger equations and their solutions, J. Australian Math. Soc. Ser. B 25, 16 (1983).

[9] N. Vishnu Priya, M. Senthilvelan, and M. Lakshmanan, Akhmediev breathers, Ma solitons, and general breathers from rogue waves: A case study in the Manakov system, Phys. Rev. E 88, 022918 (2013).

[10] B. Frisquet, B. Kibler, and G. Millot, Collision of Akhmediev Breathers in Nonlinear Fiber Optics, Phys. Rev. X 3, 041032 (2013).

[11] B. Kibler, J. Fatome, C. Finot, G. Millot, G. Genty, B. Wetzel, N. Akhmediev, F. Dias, and J. M. Dudley, Observation of Kuznetsov-Ma soliton dynamics in optical fibre, Sci. Rep. 2, 463 (2012).

[12] D. J. Kedziora, A. Ankiewicz, and N. Akhmediev, Secondorder nonlinear Schrödinger equation breather solutions in the degenerate and rogue wave limits, Phys. Rev. E 85, 066601 (2012).

[13] D. R. Solli, C. Ropers, P. Koonath, and B. Jalali, Optical rogue waves, Nature (London) 450, 1054 (2007).

[14] M. Onorato, S. Residori, U. Bortolozzo, A. Montina, and F. T. Arecchie, Rogue waves and their generating mechanisms in different physical contexts, Phys. Rep. 528, 47 (2013).

[15] N. Akhmediev, B. Kibler, F. Baronio, M. Belić, W.-P. Zhong, Y. Zhang, W. Chang, J. M. Soto-Crespo, P. Vouzas, P. Grelu, C. Lecaplain, K. Hammani, S. Rica, A. Picozzi, M. Tlidi, K. Panajotov, A. Mussot, A. Bendahmane, P. Szriftgiser, G. Genty, J. Dudley, A. Kudlinski, A. Demircan, U. Morgner, S. Amiraranashvili, C. Bree, G. Steinmeyer, C. Masoller, N. G. R. Broderick, A. F. J. Runge, M. Erkintalo, S. Residori, U. Bortolozzo, F. T. Arecchi, S. Wabnitz, C. G. Tiofack, S. Coulibaly, and M. Taki, Roadmap on optical rogue waves and extreme events, J. Opt. 18, 063001 (2016).

[16] Y. Ohta and J. K. Yang, Rogue waves in the Davey-Stewartson I equation, Phys. Rev. E. 86, 036604 (2012).

[17] A. Ankiewicz, J. M. Soto-Crespo, and N. Akhmediev, Rogue waves and rational solutions of the Hirota equation, Phys. Rev. E 81, 046602 (2010).

[18] U. Bandelow and N. Akhmediev, Persistence of rogue waves in extended nonlinear Schrödinger equations: Integrable SasaSatsuma case, Phys. Lett. A 376, 1558 (2012).
[19] F. Baronio, A. Degasperis, M. Conforti, and S. Wabnitz, Solutions of the Vector Nonlinear Schrödinger Equations: Evidence for Deterministic Rogue Wave, Phys. Rev. Lett. 109, 044102 (2012).

[20] M. Lakshmanan, K. Porsezian, and M. Daniel, Effect of discreteness on the continuum limit of the Heisenberg spin chain, Phys. Lett. A 133, 483 (1988).

[21] K. Porsezian, M. Daniel, and M. Lakshmanan, On the integrability aspects of the one-dimensional classical continuum isotropic biquadratic Heisenberg spin chain, J. Math. Phys. 33, 1807 (1992).

[22] S. M. Hoseini and T. R. Marchant, Solitary wave interaction and evolution for a higher-order Hirota equation, Wave Motion 44, 92 (2006).

[23] K. Porsezian, Completely integrable nonlinear Schrödinger type equations on moving space curves, Phys. Rev. E 55, 3785 (1997).

[24] A. Ankiewicz, D. J. Kedziora, A. Chowdury, U. Bandelow, and N. Akhmediev, Infinite hierarchy of nonlinear Schrödinger equations and their solutions, Phys. Rev. E 93, 012206 (2016).

[25] D. J. Kedziora, A. Ankiewicz, A. Chowdury, and N. Akhmediev, Integrable equations of the infinite nonlinear Schrödinger equation hierarchy with time variable coefficients, Chaos $\mathbf{2 5}$, 103114 (2015).

[26] A. Chowdury, A. Ankiewicz, and N. Akhmediev, Periodic and rational solutions of modified Korteweg-de Vries equation, Eur. Phys. J. D 70, 104 (2016).

[27] J. S. He, L. H. Wang, L. J. Li, K. Porsezian, and R. Erdélyi, Few-cycle optical rogue waves: Complex modified Korteweg-de Vries equation, Phys. Rev. E 89, 062917 (2014).

[28] Handbook of Mathematical Functions with Formulas, Graphs, and Mathematical Tables, edited by M. Abramowitz and I. A. Stegun (Dover, New York, 1972), pp. 555-566.

[29] A. Ankiewicz, Y. Wang, S. Wabnitz, and N. Akhmediev, Extended nonlinear Schrödinger equation with higher-order odd and even terms and its rogue wave solutions, Phys. Rev. E 89, 012907 (2014).

[30] A. Ankiewicz, D. J. Kedziora, and N. Akhmediev, Rogue wave triplets, Phys. Lett. A 375, 2782 (2011).

[31] D. J. Kedziora, A. Ankiewicz, and N. Akhmediev, Classifying the hierarchy of nonlinear-Schrödinger-equation rogue-wave solutions, Phys. Rev. E 88, 013207 (2013).

[32] P. Dubard, P. Gaillard, C. Klein, and V. B. Matveev, On multirogue wave solutions of the NLS equation and positon solutions of the KdV equation, Eur. Phys. J. Spec. Top. 185, 247 (2010).

[33] P. Gaillard, Families of quasi-rational solutions of the NLS equation and multi-rogue waves, J. Phys. A 44, 435204 (2011).

[34] K. B. Dysthe, Note on the modification of the nonlinear Schrödinger equation for application to deep water waves, Proc. R. Soc. A 369, 105 (1979).

[35] Yu. V. Sedletskii, The fourth-order nonlinear Schrödinger equation for the envelope of Stokes waves on the surface of a finite-depth fluid, Zh. Eksp. Teor. Fiz. 124, 200 (2003) [J. Exp. Theor. Phys. 97, 180 (2003)].

[36] A. V. Slunyaev, A high-order nonlinear envelope equation for gravity waves in finite-depth water, Zh. Eksp. Teor. Fiz. 128, 1061 (2005) [J. Exp. Theor. Phys. 101, 926 (2005)].

[37] T. Motzkin, Relations between hypersurface cross ratios, and a combinatorial formula for partitions of a polygon, for permanent preponderance, and for nonassociative products, Bull. Amer. Math. Soc. 54, 352 (1948). 